\title{
Radiomics predict postoperative survival of patients with primary liver cancer with different pathological types
}

\author{
Jiahui Zhang ${ }^{1,2}$, Xiaoli Wang ${ }^{1}$, Lixia Zhang ${ }^{1}$, Linpeng Yao ${ }^{1}$, Xing Xue ${ }^{1}$, Siying Zhang ${ }^{1}$, Xin ${ }^{3}$, \\ Yuanjun Chen ${ }^{3}$, Peipei Pang ${ }^{3}$, Dongdong Sun ${ }^{4}$, Juan $\mathrm{Xu}^{4}$, Yanjun Shi ${ }^{5}$, Feng Chen ${ }^{1}$ \\ ${ }^{1}$ Department of Radiology, The First Affiliated Hospital, Zhejiang University School of Medicine, Hangzhou, China; ${ }^{2}$ Department of Radiology, \\ Hangzhou Third Hospital, Hangzhou, China; ${ }^{3}$ GE China Medical Life Sciences Division Core Image Senior Application Team, Guangzhou, China; \\ ${ }^{4}$ Medical Big Data, AliHealth, Hangzhou, China; ${ }^{5}$ Department of Hepatobiliary and Pancreas Surgery, The Second Affiliated Hospital, Zhejiang \\ University School of Medicine, Hangzhou, China \\ Contributions: (I) Conception and design: J Zhang, X Wang, D Sun, L Zhang; (II) Administrative support: None; (III) Provision of study materials \\ or patients: None; (IV) Collection and assembly of data: J Zhang, L Yao, X Xue, S Zhang; (V) Data analysis and interpretation: All authors; (VI) \\ Manuscript writing: All authors; (VII) Final approval of manuscript: All authors. \\ Correspondence to: Feng Chen. Department of Radiology, The First Affiliated Hospital, Zhejiang University School of Medicine, Hangzhou, China. \\ Email: chenfenghz@zju.edu.cn; Yanjun Shi. Department of Hepatobiliary and Pancreas Surgery, The Second Affiliated Hospital, Zhejiang University \\ School of Medicine, Hangzhou, China. Email: shiyanjunss@hotmail.com.
}

Background: Radiomics can be used to determine the prognosis of liver cancer, but it might vary among cancer types. This study aimed to explore the clinicopathological features, radiomics, and survival of patients with hepatocellular carcinoma (HCC), mass-type cholangiocarcinoma (MCC), and combined hepatocellularcholangiocarcinoma (CHCC).

Methods: This was a retrospective cohort study of patients with primary liver cancer operated at the department of hepatobiliary surgery of the First Affiliated Hospital of Zhejiang University from 07/2013 to $11 / 2015$. All patients underwent preoperative liver enhanced MRI scans and diffusion-weighted imaging (DWI). The radiomics characteristics of DWI and the enhanced equilibrium phase (EP) images were extracted. The mRMR (minimum redundancy maximum relevance) was applied to filter the parameters.

Results: There were 44 patients with MCC, 59 with HCC, and 33 with CHCC. Macrovascular invasion, tumor diameter, positive ferritin preoperatively, positive AFP preoperatively, positive CEA preoperatively, Correlation, Inverse Difference Moment, and Cluster Prominence in model A (DWI and clinicopathological parameters) were independently associated with overall survival (OS) $(\mathrm{P}<0.05)$. Lymphadenopathy, gender, positive ferritin preoperatively, positive AFP preoperatively, positive CEA preoperatively, Uniformity, and Cluster Prominence in model B (EP and clinicopathological parameters) were independently associated with OS $(\mathrm{P}<0.05)$. Macrovascular invasion, lymphadenopathy, gender, positive ferritin preoperatively, positive CEA preoperatively, Uniformity_EP, GLCMEnergy_DWI, and Cluster Prominence_EP in model C (image texture and clinicopathological parameters) were independently associated with $\mathrm{OS}(\mathrm{P}<0.05)$. Those factors were used to construct three nomograms to predict OS.

Conclusions: Clinicopathological and radiomics features are independently associated with the OS of patients with primary liver cancer.

Keywords: Hepatocellular carcinoma (HCC); cholangiocarcinoma; radiomics; magnetic resonance imaging (MRI); postoperative survival

Submitted Dec 26, 2019. Accepted for publication Jun 05, 2020.

doi: $10.21037 /$ atm-19-4668

View this article at: http://dx.doi.org/10.21037/atm-19-4668 


\section{Introduction}

Primary liver cancer can be classified as intrahepatic cholangiocellular carcinoma, hepatocellular carcinoma (HCC), and combined hepatocellular-cholangiocarcinoma (CHCC) (1). Mass-type cholangiocarcinoma (MCC) is the main subtype of intrahepatic cholangiocarcinoma (2). CHCC has the lowest frequency among primary liver cancers, accounting for $0.4-14.2 \%$ (3). Currently, the prognosis of liver cancer is based on clinical and biochemical features such as HBeAg, AFP, HBV-DNA load, prothrombin, creatinine, hypertension, statin treatment, cirrhosis, tumor number, tumor size, encapsulation, microvascular invasion, and surgical margins (4-6), but their prognostic value is limited.

The prognosis of CHCC is worse than that of HCC and cholangiocarcinoma, and survival is lower $(7,8)$. Nevertheless, the prognosis of CHCC in relation to HCC and cholangiocarcinoma may vary (9). It has been suggested that vascular invasion, tumor size, tumor location, tumor multifocal, tumor stage, and lymph node metastasis are key factors affecting the prognosis of CHCC $(10,11)$, while another study suggested that high levels of $\alpha$-fetoprotein (AFP) ( $\geq 400 \mathrm{IU} / \mathrm{L})$ is an independent factor affecting the prognosis of CHCC (10).

Texture analysis (TA) of tumor images can be objectively measured and has a high value in the characterization, evaluation of therapeutic effects, and prognosis of tumors $(12,13)$. In lung cancer, radiomics characterization is an independent biomarker for assessing the disease-free survival (DFS) of patients with early non-small cell lung cancer (14).

The radiomics signatures that could predict the prognosis of liver cancer still remain to be defined. Hence, this retrospective cohort study explored the key factors affecting survival after radical surgery for liver cancer, by analyzing the clinicopathological features, radiomics, and survival of three different pathological types of primary liver cancer, i.e., HCC, MCC, and CHCC. We present the following article in accordance with the STROBE reporting checklist (available at http://dx.doi.org/10.21037/atm-194668).

\section{Methods}

This retrospective cohort study was approved by the institutional review board of the First Affiliated Hospital of Zhejiang University (No.: 2017-712). Informed consent was waived because of the retrospective nature of the study.

\section{Patients}

Patients with primary liver cancer operated at the department of hepatobiliary surgery of the First Affiliated Hospital of Zhejiang University from July 2013 to November 2015 were included. The inclusion criteria were: (I) complete resection of the primary liver cancer; (II) MCC, HCC, or CHCC confirmed by surgical pathology; (III) without distant metastasis; (IV) preoperative magnetic resonance imaging (MRI) and contrast-enhanced MRI performed on a 3.0-T Signa HDxt (GE Healthcare, Waukesha, WI, USA); and (V) available follow-up data. The exclusion criteria were: (I) significant MRI artifacts affecting diagnosis; (II) lesions $\leq 5 \mathrm{~mm}$, affecting image texture feature extraction; (III) incomplete clinical data; (IV) received liver transplantation; (V) recurrence of liver cancer; or (VI) lost to follow-up or incomplete data.

\section{Clinicopathological data}

The clinical data were extracted (by JZ) from the medical charts. All patients had a detailed clinical history and a complete physical examination. Postoperative tumor pathology was assessed by two experienced pathologists to give a final diagnosis. The patients were grouped based on the original histopathological diagnosis.

\section{MRI parameters}

MRI was performed using a 3.0-T Signa HDxt (GE Healthcare, Waukesha, WI, USA) MRI system and an 8-channel phased-array surface coils. The parameters of the breath-hold T1WI pressure-sensitive LIVA scan sequence was: repetition time (TR)/echo time (TE) $(3.1 / 1.5 \mathrm{~ms})$, matrix $320 \times 256$, layer thickness $5 \mathrm{~mm}$, and field-of-view (FOV) $40 \times 32 \mathrm{~cm}$. The intravenous injection of $0.1 \mathrm{mmol} /$ $\mathrm{kg}$ of the contrast agent Gd-DTPA was performed using a high-pressure syringe at $2.5 \mathrm{~mL} / \mathrm{s} ; 15 \mathrm{~mL}$ of normal saline were injected at the same rate. The arterial phase, portal phase, and equilibration phase (EP) liver images were collected after 20,60, and $150 \mathrm{~s}$ of contrast media injection, respectively. The scanning parameters of diffusion-weighted imaging (DWI) were: TR 9,000 ms, TE $60.5 \mathrm{~ms}$, FOV $3,828.5 \mathrm{~cm}$, matrix $128 \times 128$, layer thickness $5 \mathrm{~mm}$, and diffusion coefficient b value $1,000 \mathrm{~s} / \mathrm{mm}^{2}$.

\section{Image analysis}

Analysis of the target area heterogeneity was performed 
using dedicated AK software (Analysis Kit, GE Healthcare, Waukesha, WI, USA) to obtain a series of image features. All magnetic resonance enhanced EP images (DICOM format) containing liver lesions were imported into the $\mathrm{AK}$ software. All layers of all lesions were manually delineated as regions of interest (ROIs) (15) and segmented by an attending radiologist with 10 years of work experience. The software automatically combined the lesions at all levels and calculated the quantitative image feature parameters in the ROI (Figure S1). Considering that the three types of primary liver cancer have different enhancement features, EP was chosen because all lesions have the clearest images and display limited variability among them.

\section{Radiomics}

The minimum redundancy maximum relevance (mRMR) method and the elastic network algorithm were used to select the 384 feature parameters and DWI-acquired image features. If there was a missing value in the image feature column, the missing value was filled with the mean of the column. Five parameters remained after mRMR de-redundancy and elastic network dimension reduction (Correlation_angle90_offset1, Quantile0.975, Correlation_angle45_offset7, Inverse Difference Moment_ All Direction_offset4_SD, and Cluster Prominence_ angle0_offset 7$)$. In addition, 384 image features were acquired from the EP. If there was a missing value in the image feature column, the missing value was filled with the mean of the column. Five parameters remained after mRMR de-redundancy and elastic network dimension reduction (Max Intensity, Haralick Correlation_angle135_ offset7, uniformity, Cluster Prominence_angle135_offset7, and Inertia_angle135_offset7). The DWI and EP each acquired 384 image features. If there was a missing value in the image feature column, the missing value was filled with the mean of the column, and seven parameters remained after mRMR de-redundancy and elastic network dimension reduction (Haralick Correlation_angle135_offset7_EP, Correlation_angle45_offset7_DWI, uniformity_EP, Max Intensity_EP, GLCM Energy_angle0_offset7_DWI, Cluster Prominence_angle0_offset7_DWI, and Cluster Prominence_angle135_offset7_EP).

\section{Follow-up}

The patients were followed by routine outpatient examination until death or November 30, 2017. Overall survival (OS) was the time from surgery to death.

\section{Statistical analysis}

There was no sample size calculation as all eligible patients during the study period were included. Statistical analysis was performed using R (glmnet package, cv.glmnet function). Continuous variables are presented as means \pm standard deviation and were analyzed using analysis of variance (ANOVA) with the least significant difference (LSD) post hoc test. Categorical variables are expressed as frequencies and were analyzed using the chi-square test, Fisher's exact test, or Kruskal-Wallis test. Because of the large differences in the numerical range between quantitative feature parameters of radiomics, the standard score method (z-score) was used to standardize the variables. Because of the redundancy between the quantitative image feature parameters of tumor lesions, the mRMR feature selection algorithm was used to calculate the correlation and redundancy between features, and then the elastic network algorithm was applied. The feature parameters were selected using the 5-fold cross-validation method. OS is presented as median (range) and Kaplan-Meier survival curves and was analyzed using the log-rank test at 6 months, 1 year, and 3 years. Four multivariable Cox regression models were established to determine the prognostic factors. The selection of the parameters to be included was based on the significance in the univariable analyses, as well as on the known clinical significance of parameters, according to the literature and the authors' experience. All clinical indicators were tested for collinearity. The four models were: Model A, DWI, and clinicopathological features; Model B, EP, and clinicopathological features; and Model C, DWI, EP, and clinicopathological features; Model D, clinicopathological features only. Based on the results of the factors affecting the OS, four nomograms were established, and a decision curve analysis was performed in order to determine the clinical net benefit of those nomograms. Two-sided $\mathrm{P}$ values $<0.05$ were considered statistically significant.

\section{Results}

\section{Characteristics of the patients}

There were 44 patients with MCC (45 lesions, one patient had two lesions), 59 with HCC (59 lesions), and 33 with CHCC (36 lesions, one patient had two lesions, and one patient had three lesions) (Table 1). 
Table 1 Clinicopathological features of the patients with primary hepatic carcinoma

\begin{tabular}{|c|c|c|c|c|}
\hline Variables & $\operatorname{MCC}(n=44)$ & $\mathrm{HCC}(\mathrm{n}=59)$ & $\mathrm{CHCC}(\mathrm{n}=33)$ & $P$ \\
\hline Age (years) & $58.4 \pm 9.8$ & $58.2 \pm 11.2$ & $54.4 \pm 11.3$ & 0.202 \\
\hline$>55$ years & $29(65.9 \%)$ & $36(61.0 \%)$ & $18(54.5 \%)$ & 0.605 \\
\hline Tumor number & & & & 0.130 \\
\hline 2 & $1(2.3 \%)$ & 0 & $1(3.0 \%)$ & \\
\hline 3 & 0 & 0 & $1(3.0 \%)$ & \\
\hline Macrovascular invasion & $9(20.5 \%)$ & $8(13.6 \%)$ & $1(3 \%)$ & 0.083 \\
\hline Lymphadenopathy* & $18(40.9 \%)^{a}$ & $10(16.9 \%)^{b}$ & $13(39.4 \%)^{\mathrm{ab}}$ & 0.013 \\
\hline HBsAg positive & $19(43.2 \%)^{a}$ & $44(74.6 \%)^{\mathrm{b}}$ & $27(81.8 \%)^{\mathrm{b}}$ & $<0.001$ \\
\hline CA19-9 (U/mL) & $420.83 \pm 1,073.48^{a}$ & $24.36 \pm 42.78^{b}$ & $42.67 \pm 63.68^{b}$ & 0.003 \\
\hline CA19-9 >37 U/mL & $14(31.8 \%)^{\mathrm{a}}$ & $2(3.4 \%)^{b}$ & $6(18.2 \%)^{a b}$ & $<0.001$ \\
\hline CA125 (U/mL) & $153.6 \pm 736.66$ & $14.87 \pm 16.17$ & $19.66 \pm 33.61$ & 0.208 \\
\hline $\mathrm{CA} 125>35 \mathrm{U} / \mathrm{mL}$ & $8(18.2 \%)^{a}$ & $2(3.4 \%)^{b}$ & $2(6.1 \%)^{\mathrm{ab}}$ & 0.026 \\
\hline Ferritin $(\mathrm{ng} / \mathrm{mL})$ & $210.55 \pm 160.98$ & $295.97 \pm 369.52$ & $229.78 \pm 159.2$ & 0.252 \\
\hline Ferritin $>323$ ng/mL & $8(18.2 \%)$ & $14(23.7 \%)$ & $9(27.3 \%)$ & 0.631 \\
\hline $\operatorname{AFP}(\mathrm{ng} / \mathrm{mL})$ & $8.59 \pm 25.72$ & $2,399.39 \pm 8,286.27$ & $290.62 \pm 644.95$ & 0.059 \\
\hline
\end{tabular}

*, lymphadenopathy was assessed by magnetic resonance imaging; ${ }^{a, b}$, different letters indicate statistically significant differences. HCC, hepatocellular carcinoma; MCC, mass-type cholangiocarcinoma; CHCC, combined hepatocellular-cholangiocarcinoma; AFP, $\alpha$-fetoprotein; HBsAg, hepatitis surface antigen; CA199, carbohydrate antigen 19-9; CA125, carbohydrate antigen 125; CEA, carcinoembryonic antigen.

\section{Radiomics features}

The optimized lambdas $(\lambda)$ of MRI, EP, and DWI were $0.04608123,0.06285455$, and $0.06285455[\ln (\lambda):-3.077349$, -2.766932 , and -2.766932], respectively (Figure S2).

\section{OS according to the pathological types of primary liver cancer}

The median OS of MCC, HCC, and CHCC was 29.8 (1.7-50.3), 30.8 (1.0-52.7), and 25.5 (1.0-51.3) months, respectively. The OS rates at 6 months, 1 year, and 3 years were $88.6 \%$ (39/44), 86.4\% (38/44), and 63.6\% (28/44); $94.9 \%$ (56/59), 89.8\% (53/59), and 76.3\% (45/59); and $96.9 \%(32 / 33), 81.8 \%(27 / 33)$, and $48.5 \%(16 / 33)$, respectively $(\mathrm{P}=0.020)$. The differences in $\mathrm{OS}$ were significant between HCC and CHCC $(\mathrm{P}=0.005)$ (Figure 1).

\section{Multivariable analysis and nomogram of OS}

Macrovascular invasion, tumor size, positive ferritin preoperatively, positive AFP preoperatively, positive CEA preoperatively, Correlation, Inverse Difference Moment, and Cluster Prominence in model A (five DWI feature parameters and 12 clinicopathological features) and were independent risk factors for OS $(\mathrm{P}<0.05)$ (Table 2). Lymphadenopathy, gender, positive ferritin preoperatively, 


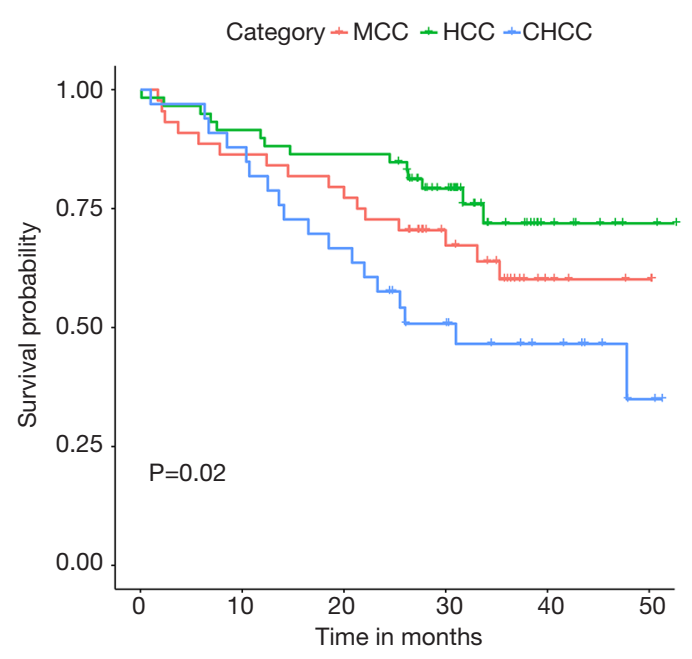

Figure 1 Kaplan-Meier curves of overall survival of mass-type cholangiocarcinoma (MCC) $(n=44)$, hepatocellular carcinoma (HCC) (n=59), and combined hepatocellular-cholangiocarcinoma (CHCC) ( $n=33)$. The three groups of different types of primary liver cancer patients had significant differences in survival $(\mathrm{P}=0.02)$. There was a significant difference between the HCC and CHCC groups $(\mathrm{P}=0.005)$.

Table 2 Elastic net and multivariable Cox model analysis of OS for primary hepatic carcinomas using a combination of clinicopathological features and texture parameters derived from MR-DWI

\begin{tabular}{lcc}
\hline \multirow{2}{*}{ Variables } & \multicolumn{2}{c}{ Overall survival } \\
\cline { 2 - 3 } & $\mathrm{HR}(95 \% \mathrm{Cl})$ & $\mathrm{P}$ \\
\hline Macrovascular invasion* & $0.296(0.099-0.882)$ & 0.029 \\
$\begin{array}{l}\text { The largest tumor diameter } \\
\leq 3 \mathrm{~cm}^{*}\end{array}$ & $1.413(1.150-1.736)$ & $<0.001$ \\
Ferritin $>323 \mathrm{ng} / \mathrm{mL}$ & $2.484(1.073-5.751)$ & 0.034 \\
AFP $>20 \mathrm{ng} / \mathrm{mL}$ & $2.005(1.017-3.951)$ & 0.045 \\
CEA $>5$ ng/mL & $5.372(1.871-15.423)$ & 0.002 \\
Correlation_angle90_offset1 & $0.198(0.071-0.553)$ & 0.002 \\
$\begin{array}{l}\text { Inverse Difference Moment_ } \\
\text { AllDirection_offset4 }\end{array}$ & $1.501(1.061-2.124)$ & 0.022 \\
$\begin{array}{l}\text { Cluster Prominence_ } \\
\text { angle0_offset7 }\end{array}$ & $0.183(0.071-0.471)$ & 0.001 \\
\hline
\end{tabular}

*, macrovascular invasion, tumor size, and lymphadenopathy were assessed by magnetic resonance imaging. AFP, $\alpha$-fetoprotein; HBsAg, hepatitis surface antigen; CA199, carbohydrate antigen 19-9; CA125, carbohydrate antigen 125; CEA, carcinoembryonic antigen; OS, overall survival; HR, hazard ratio. positive AFP preoperatively, positive CEA preoperatively, Uniformity, and Cluster Prominence in model B (five EP feature parameters +12 clinicopathological features) and independent risk factors affecting OS $(\mathrm{P}<0.05)$ (Table 3). Macrovascular invasion, lymphadenopathy, gender, positive ferritin preoperatively, positive CEA preoperatively, Uniformity_EP, GLCMEnergy_DWI, and Cluster Prominence_EP in model C (seven image texture feature parameters + 12 clinicopathological features) and independent risk factors affecting OS $(\mathrm{P}<0.05)$ (Table 4). Table 5 shows the model that only includes the clinicopathological variables. Gender, lymphadenopathy, the largest tumor diameter, ferritin, AFP, and CEA were independent risk factors of OS. These factors were used to construct three nomograms (Figure 2). By calculating the total area under the curve, the net benefit of nomogram c (blue line) was significantly higher than the nomograms a (red line), b (green line) and d (yellow) (Figure 3). The supplementary model with clinicopathological variables only had the lowest net benefit (yellow line) globally.

\section{Discussion}

This study aimed to explore the clinicopathological features, radiomics, and survival of patients with HCC, MCC, and CHCC. The results suggest that clinicopathological and radiomics features are independently associated with the OS of patients with primary liver cancer.

The histopathological features of HCC and CHCC are completely different, and their prognosis is also different, but the prognostic factors associated with those tumors are inconsistent. Whether radiomics could be helpful for prognosis needs to be verified. Many authors suggest that the prognosis of CHCC is poorer than that of HCC. Maeda et al. (16) showed that the clinical background of CHCC and HCC is similar, but that CHCC has poor prognosis and survival compared with HCC because of its higher metastatic potential. Consistently, in the present study, the median survival of the patients was 25.5 months, significantly lower than for those with HCC (30.8 months). The survival of the CHCC group was lower than that of the other two groups, while the survival of the patients with MCC was similar to those with HCC. The multivariable Cox regression model revealed that elevated preoperative AFP, ferritin, and CEA levels, tumor size, macrovascular invasion, lymphadenopathy, gender, Correlation, Inverse Difference Moment, Cluster Prominence, Uniformity, and 
Table 3 Elastic net and multivariable Cox model analysis of OS for primary hepatic carcinomas using a combination of clinicopathological features and texture parameters derived from MR-EP

\begin{tabular}{lcc}
\hline \multirow{2}{*}{ Variables } & \multicolumn{2}{c}{ Overall survival } \\
\cline { 2 - 3 } & $\mathrm{HR}(95 \% \mathrm{Cl})$ & $\mathrm{P}$ \\
\hline Gender, male & $0.394(0.175-0.889)$ & 0.025 \\
Lymphadenopathy & $2.197(1.074-4.494)$ & 0.031 \\
Ferritin $>323 \mathrm{ng} / \mathrm{mL}$ & $2.639(1.210-5.753)$ & 0.015 \\
AFP $>20 \mathrm{ng} / \mathrm{mL}$ & $2.351(1.175-4.704)$ & 0.016 \\
CEA $>5 \mathrm{ng} / \mathrm{mL}$ & $2.885(1.066-7.809)$ & 0.037 \\
Max intensity & $1.044(0.723-1.506)$ & 0.818 \\
Uniformity & $0.453(0.276-0.745)$ & 0.002 \\
$\begin{array}{l}\text { Cluster Prominence_ } \\
\text { angle135_offset7 }\end{array}$ & $0.544(0.331-0.895)$ & 0.017 \\
\hline
\end{tabular}

*, used to mark the corresponding variables were evaluated by magnetic resonance imaging. AFP, $\alpha$-fetoprotein; HBsAg, hepatitis surface antigen; CA199, carbohydrate antigen 199; CA125, carbohydrate antigen 125; CEA, carcinoembryonic antigen; OS, overall survival; HR, hazard ratio.

Table 4 Elastic net and multivariable Cox model analysis of OS for primary hepatic carcinomas using a combination of clinicopathological features and texture parameters derived from MR-DWI and MR-EP

\begin{tabular}{lcc}
\hline \multirow{2}{*}{ Variables } & \multicolumn{2}{c}{ Overall survival } \\
\cline { 2 - 3 } & $\mathrm{HR}(95 \% \mathrm{Cl})$ & $\mathrm{P}$ \\
\hline Gender, male & $0.375(0.161-0.873)$ & 0.023 \\
Macrovascular invasion* & $0.297(0.096-0.921)$ & 0.036 \\
Lymphadenopathy* & $2.337(1.130-4.830)$ & 0.022 \\
AFP $>20$ ng/mL & $2.671(1.348-5.293)$ & 0.005 \\
CEA >5 ng/mL & $4.112(1.390-12.162)$ & 0.011 \\
Uniformity_EP & $0.420(0.253-0.695)$ & $<0.001$ \\
$\begin{array}{l}\text { GLCMEnergy_angle0_ } \\
\text { offset7_DWI }\end{array}$ & $1.550(1.167-2.060)$ & 0.002 \\
$\begin{array}{l}\text { ClusterProminence_ } \\
\text { angle135_offset7_EP }\end{array}$ & $0.562(0.335-0.943)$ & 0.029 \\
\hline \multirow{2}{*}{ used to mark the corresponding variables were evaluated }
\end{tabular}

*, used to mark the corresponding variables were evaluated by magnetic resonance imaging. AFP, $\alpha$-fetoprotein; HBsAg, hepatitis surface antigen; CA199, carbohydrate antigen 199; CA125, carbohydrate antigen 125; CEA, carcinoembryonic antigen; OS, overall survival; HR, hazard ratio.
Table 5 Multivariable Cox model analysis of OS for primary hepatic carcinomas using clinicopathological features

\begin{tabular}{lcc}
\hline \multirow{2}{*}{ Variables } & \multicolumn{2}{c}{ Overall survival } \\
\cline { 2 - 3 } & $\mathrm{HR}(95 \% \mathrm{Cl})$ & $\mathrm{P}$ \\
\hline Gender, male & $0.383(0.177-0.828)$ & 0.015 \\
Lymphadenopathy & $2.158(1.090-4.273)$ & 0.027 \\
The largest tumor diameter & $1.328(1.158-1.532)$ & $<0.001$ \\
$\leq 3 \mathrm{~cm}^{*}$ & & \\
Ferritin $>323 \mathrm{ng} / \mathrm{mL}$ & $2.417(1.134-5.153)$ & 0.022 \\
AFP $>20 \mathrm{ng} / \mathrm{mL}$ & $3.045(1.551-5.978)$ & 0.001 \\
CEA $>5 \mathrm{ng} / \mathrm{mL}$ & $2.790(1.059-7.355)$ & 0.038 \\
\hline
\end{tabular}

*, used to mark the corresponding variables were evaluated by magnetic resonance imaging. AFP, $\alpha$-fetoprotein; HBsAg, hepatitis surface antigen; CA199, carbohydrate antigen 199; CA125, carbohydrate antigen 125; CEA, carcinoembryonic antigen; OS, overall survival; HR, hazard ratio.

GLCMEnergy were independently associated with OS of the three groups of primary liver cancer after operation. Three nomograms were constructed. The evaluation of the survival of the three groups of primary liver cancer by DWI, EP, and clinicopathological features group was the best. Therefore, radiomics features (ClusterProminence, Uniformity, and GLCMEnergy) probably have great medical value in the evaluation of postoperative OS of patients with primary liver cancer.

AFP is the first biological indicator and the most widely used in the diagnosis, prognosis, and monitoring of HCC. Cucchetti et al. (17) found that preoperative AFP levels in patients with HCC were significantly associated with early recurrence. The occurrence of AFP positivity in patients with cholangiocarcinoma is low, and about $20-25 \%$ of patients with intrahepatic cholangiocarcinoma have serum AFP levels $>20 \mathrm{ng} / \mathrm{mL}$ (18). Ishikawa et al. (19) reported one case of AFP-positive intrahepatic cholangiocarcinoma and found that the cancer cells contained some stem cell markers. Ishii et al. (20) showed that AFP-positive intrahepatic cholangiocarcinoma possesses stem-like cells. Park et al. (21) suggested that high levels of AFP are independently associated with the prognosis of CHCC. We found that two patients (4.5\%), 29 patients (49.2\%), and 20 patients $(60.6 \%)$ were found to have higher than normal AFP levels in the MCC, HCC, and CHCC groups. Asayama et al. (22) showed that AFP levels are similar in 
A
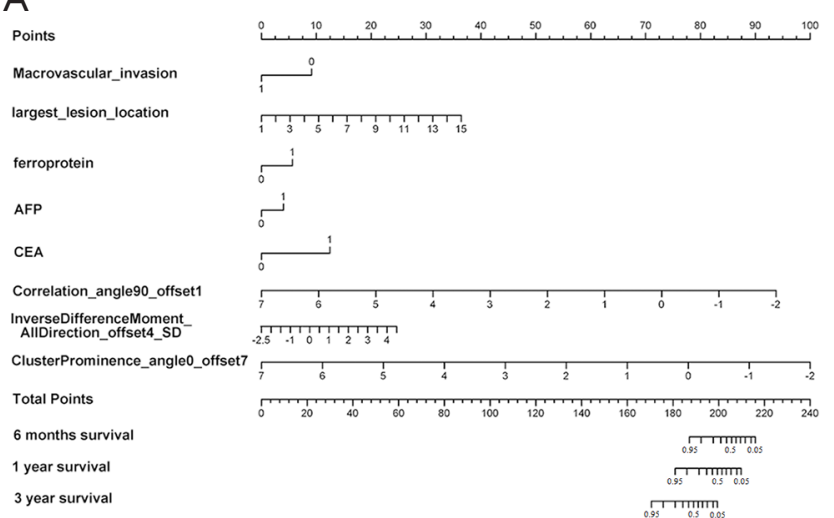

-2.5
$1+1$

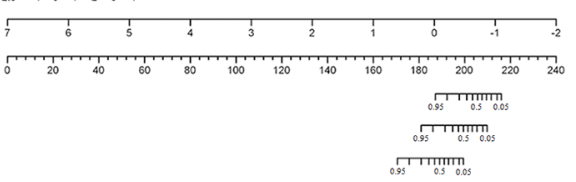

C
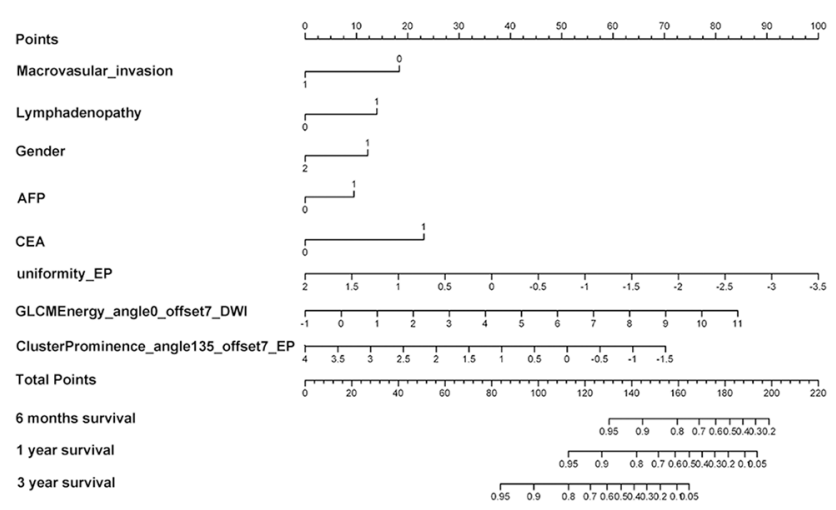

B

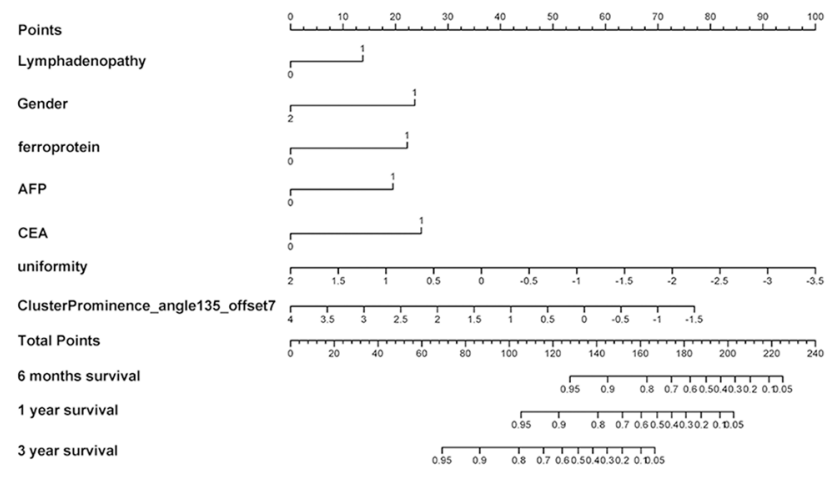

D

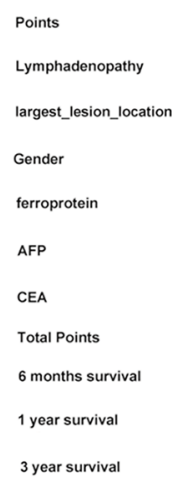

Figure 2 Nomogram for predicting overall survival (OS) of patients who received liver resection for mass-type cholangiocarcinoma (MCC), hepatocellular carcinoma (HCC), and combined hepatocellular-cholangiocarcinoma (CHCC). (A) Clinicopathological features and texture parameters calculated from diffusion-weighted imaging (DWI), (B) clinicopathological features and texture parameters calculated from the equilibrium phase (EP); (C) clinicopathological features and texture parameters calculated from DWI and EP; (D) clinicopathological features only. AFP, $\alpha$-fetoprotein; CEA, carcinoembryonic antigen.

patients with HCC and CHCC, and are hepatocellularderived, while Lee et al. (23) suggested that the levels of AFP in CHCC is lower than that in HCC. The results of this study are slightly different from the above reports. Indeed, the positive rate of AFP in CHCC was significantly higher than in HCC. The OS of CHCC was lower than that of HCC, which was positively correlated with the AFP positive rate.

Ferritin is an intracellular protein that stores iron and plays a key role in iron homeostasis. Increased serum ferritin levels are seen in patients with increased iron stores, necrotizing liver inflammation, and systemic inflammatory response (24). Hann et al. (25) showed that elevated levels of ferritin in patients with chronic liver disease was a risk factor for HCC and that, similar to AFP, it can be used as a serum marker for the evaluation of HCC. Uchino et al. (26) found that extremely high or low serum ferritin levels were an independent risk factor for HCC in men with chronic hepatitis C. The use of ferritin as a biomarker to evaluate the survival to liver cancer has not been reported in the literature, but it has been reported as an independent prognostic factor in other areas. For example, Urano et al. (27) found that sorafenib treatment can prolong the OS of patients with low iron status. Sorafenib and deferasirox reduce iron content and may become a new type of treatment for HCC combined with chemotherapy. Weismuller et al. (28) showed that serum ferritin levels could predict the mortality rate of patients waiting for liver transplantation and death after liver transplantation. In the present study, 8 (20.5\%), 14 (23.7\%), and 9 patients (27.3\%) 


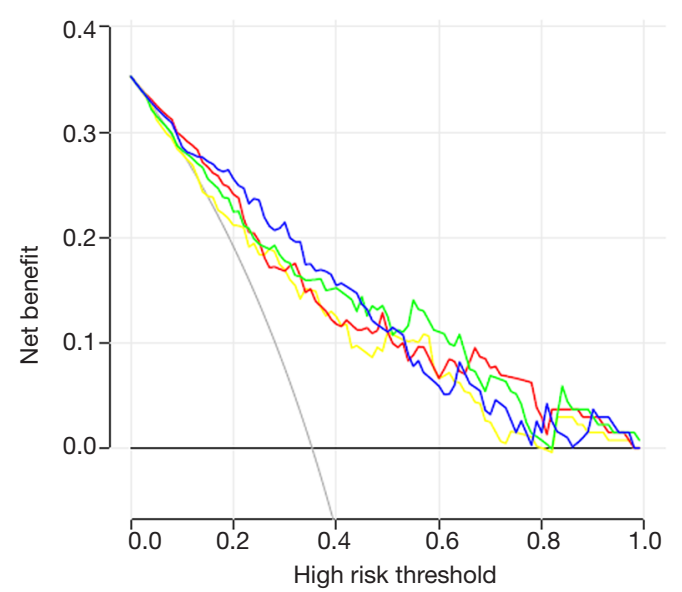

Figure 3 Analysis of imaging features and clinical and pathological features by a decision curve nomogram. The red, green, blue and yellow lines represent the nomograms from models 1, 2, 3 and 4 respectively. The light gray line represents the hypothesis that all clinicopathological and imaging features are related to postoperative survival time. The dark gray line indicates that there is no hypothesis that all clinicopathological and imaging features are related to postoperative survival.

had higher than normal ferritin levels in the MCC, HCC, and CHCC groups, without significant differences among the three groups. Furthermore, the multivariable Cox regression models showed that ferritin was independently associated with OS in the three groups of primary liver cancers, indicating that higher positive rates of ferritin were associated with shorter OS. The reason may be that ferritin increases liver load and liver necrotizing inflammatory response in patients with liver cancer, resulting in shortened survival.

CEA is a glycoprotein derived from the endoderm. CEA elevation can be seen in various digestive tumors such as cholangiocarcinoma, gastric cancer, colon cancer, and pancreatic cancer. Konomi et al. (29) showed that the CEA levels are helpful to distinguish between benign and malignant liver and biliary diseases, and higher CEA values indicate that there are malignant tumors besides the remaining common bile duct stones, usually pancreatic head cancer or cancer of the duodenum ampulla. In liverrelated tumors, CEA is highly expressed in metastatic liver cancer, cholangiocarcinoma, and CHCC, but is mostly negative in HCC. Juntermanns et al. (30) showed that the OS of patients with cholangiocarcinoma and high CEA serum levels before the operation was lower than that of patients with lower CEA serum levels and that the unresectable rate of these patients was significantly higher. According to a previous study, only CEA, not CA19-9, is an independent predictor of survival after surgery, and CEA may help identify patients with bile duct cell carcinoma with poor prognosis after tumor resection (31). In the present study, there were six $(13.6 \%)$, three $(5.1 \%)$, and three patients $(9.1 \%)$ with higher than normal CEA levels in the MCC, HCC, and CHCC groups. The multivariable Cox regression model showed that CEA was an independent risk factor affecting the OS of the three groups after the operation.

The incidence of HCC is high among male patients. Gender is an independent factor affecting prognosis after hepatectomy, and the survival time of male patients is shorter than that of women (32). The male gender is related to the high lymph node metastasis rate in patients with early intrahepatic cholangiocarcinoma (33). Similarly, Lee et al. (34) showed that the prognosis of CHCC was worse than that of HCC and cholangiocarcinoma, and the recurrence time of tumor in male patients was shorter after the operation than that of females. Yang et al. (35) found in a multivariable analysis that the median OS of women with HCC was significantly longer than that of men. In the present study, the male-to-female ratios were 1.44 (26/8), 5.56 (50/9), and 1.75 (21/12) in the MCC, HCC, and CHCC groups, respectively, and the multivariable Cox regression model showed that gender was an independent risk factor for OS.

The survival and prognosis of patients with liver cancer and lymph node metastasis are significantly worse than those without lymph node metastasis. Some patients have no liver tumor recurrence after hepatectomy but die from extensive lymph node metastasis. Therefore, the TNM7 directly classifies lymph node metastasis (N1) of liver cancer into stage IVa because its survival rate is similar to that of distant metastasis (M1) (36). Lymphadenopathy in the present study was evaluated by MRI. Although lymphadenopathy and the presence of lymph node metastasis are not entirely consistent, it still reflects the lymph node metastasis of liver cancer to some extent. Park et al. (37) showed that the overall accuracy of lymph node metastasis by CT axial imaging is $57 \%$, while the accuracy of MRI was higher than that of CT. Hepatic hilar or postperitoneal lymphadenopathy in the MCC, HCC, and CHCC groups was found in 18 (40.9\%), 10 (16.9\%), and $13(39.3 \%)$, respectively. The lymphadenopathy rate of the HCC group was lower than that of the other two groups, which was consistent with OS, but there was no significant 
difference in the lymphadenopathy rate between the MCC and CHCC groups. The multivariable Cox regression model showed that lymphadenopathy was an independent risk factor for the survival of the three groups of primary liver cancer.

$\mathrm{TA}$ is the basis of image histology research. It can measure the characteristics of tissue heterogeneity that cannot be observed by the human eye and can quantitatively display the subtle changes of image pixel values and their arrangement. TA can objectively measure the unevenness of the grayscale distribution without being affected by subjective analysis, professional level, and inherent limitations of traditional medical image interpretation. Cluster prominence is a measure of the skewness and asymmetry of the intensity of horizontal distribution in an image and is inversely proportional to the amount of asymmetry in the image.

Vargas et al. (38) predicted the prognosis and survival of patients with high-grade serous ovarian cancer by delineating and segmenting the CT tumor focus of each stage before surgery. After extracting the imaging features, Cluster Prominence was significantly related to short OS and incomplete surgical resection. The average texture features of Cluster prominence_angle 135_offset 7 in MCC, HCC, and CHCC were $4.14 \times 10^{7}, 3.52 \times 10^{7}$, and $4.75 \times 10^{7}$ in the present study. The Cluster prominence in the EP of the HCC group was the lowest, indicating that the horizontal distribution intensity in HCC images was less skewed and asymmetric, which may be related to the good prognosis and survival of HCC. Uniformity is a measure of the sum of squares of each discrete value in the region of interest of the image. This is a measure of image heterogeneity, in which greater uniformity means greater heterogeneity. Bae et al. (39) suggested that the quantitative imaging values extracted from CT images of dual-energy spectrum iodine contrast maps could help predict the pathological invasion of lung adenocarcinoma. In multiple logistic regression analyses, uniformity was significantly different between lung adenocarcinoma grades II and I. In the present study, the mean value of uniformity of EP in MCC, HCC, and CHCC was $0.784,0.813$, and 0.815 . Among them, the mean value of the CHCC group was the largest, indicating that the region of interest of the CHCC image was more homogeneous or heterogeneous than in the other two groups, which may be related to the poor prognosis of CHCC. Energy reflects the uniformity of image gray distribution and texture thickness. If all values of the cooccurrence matrix are equal, the energy value is small; on the other hand, if some of these values are large and others are small, the energy value is large. Chen et al. (40) found no difference in GLCM_Energy between the false and the progression of glioblastoma. In the present study, the mean value of GLCM_Energy in MCC, HCC, and CHCC was 0.0017, 0.0009, and 0.0031, respectively. The CHCC group had the largest mean value, indicating that the difference in texture thickness of the region of interest in the image was more obvious than in the other two groups, which may be related to poor survival after surgery. Correlation is a measure of the similarity of spatial gray level co-occurrence matrix elements in the row or column direction. The mean values of texture feature (Correlation) of diffuse images in MCC, HCC, and CHCC were 0.0005, 0.0007, and 0.0004. Among them, the Correlation value in the CHCC group was the smallest, indicating that the pixel value of the image region of interest matrix is more different than that of the two other groups and may be related to the poor prognosis of the tumor. The Inverse Difference Moment reflects the homogeneity of the image texture and measures how much the image texture changes locally. Zhang et al. (41) recently showed that extracting MR texture features (T2WI sequence) before tumor treatment had the potential to predict the therapeutic response to colorectal liver metastasis, among which texture features (Correlation and Inverse Difference Moment) showed significant differences between the response and non-response groups. In the present study, the mean values of Inverse Difference Moment in MCC, HCC, and CHCC were 0.078, 0.097, and 0.067 , respectively, with the HCC group having the largest mean value, indicating a lack of change among different regions of the texture of the ROI in the image, which is more uniform locally than MCC and CHCC, and may be related to good prognosis after operation.

Radiomics is a novel field of research that mostly arose in the last decade with the development of artificial intelligence and pattern recognition tools. Radiomics is, therefore, still in its infancy. Nevertheless, the future looks good for the development of radiomics tools for precision medicine (15). At present, there is no one-toone correspondence between specific radiomics features and specific clinicopathological features. So far, research identified patterns of radiomics features with cancer survival, which is itself determined by numerous factors $(38,42-44)$. Radiomics is a new research domain, and much work still needs to be done. The advantages of radiomics have been shown for other types of cancer $(38,42,44)$. Vargas et al. (38) showed in ovarian cancer that inter-site 
similarity entropy, similarity level cluster shade, and intersite similarity level cluster prominence were associated with survival. Huang et al. (42) showed that the MRI-derived GLCM inverse difference moment normalized (IDMN) and the PET-derived GLCM cluster prominence were among the key features in the predictive models for recurrence-free survival. Lee et al. (44) showed that for the nonenhancing lesion, skewness and variance ratios of relative $\mathrm{CBV}$ texture were associated with OS. For the kinetic TA, the Haralick correlation was associated with OS. Nevertheless, data are still missing for liver cancer. Only Cozzi et al. (43) identified nine clinical and 13 radiomics features with multiple and complex cross-correlations with survival in HCC.

There are shortcomings to this study. Because it was a retrospective study from a single medical center, differences in treatment may have an impact on the results. Indeed, 20 patients with HCC received transcatheter arterial chemoembolization (TACE), while only one and two patients with MCC and CHCC received TACE. The follow-up time was short. The proportion of patients lost to follow-up is large (21/80 for patients with HCC, 29/65 for patients with CHCC, and 18/65 for patients with MCC). Cirrhosis could not be included among the analyzed parameters because this data was not consistently reported in the charts and because this feature could not be determined based on imaging in many patients. In addition, PFS was not evaluated. Of course, the results might only apply to patients with HCC, CHCC, and MCC, and not to all patients with liver cancer. In addition, the study was carried out at a single center in Chinese patients, limiting the generalizability of the results.

In conclusion, positive AFP, ferritin, and CEA preoperatively, macrovascular invasion, tumor size, gender, and some radiomics features are independently associated with postoperative OS of patients with primary liver cancer. Radiomics features such as Correlation, Inverse Difference Moment, Cluster Prominence, Uniformity, and GLCMEnergy may be potential imaging biomarkers for the postoperative OS of primary liver cancer.

\section{Acknowledgments}

Funding: This study was funded by the Zhejiang Provincial Natural Science Foundation Committee-Zhejiang Society for Mathematical Medicine Joint Fund Major Project (LSD19H180003) and the Major research plan integration project of China National Natural Science Foundation of China (91630311).

\section{Footnote}

Reporting Checklist: The authors have completed the STROBE reporting checklist. Available at http://dx.doi. org/10.21037/atm-19-4668

Data Sharing Statement: Available at http://dx.doi. org/10.21037/atm-19-4668

Conflicts of Interest: All authors have completed the ICMJE uniform disclosure form (available at http://dx.doi. org/10.21037/atm-19-4668). The authors have no conflicts of interest to declare.

Ethical Statement: The authors are accountable for all aspects of the work in ensuring that questions related to the accuracy or integrity of any part of the work are appropriately investigated and resolved. The study was conducted in accordance with the Declaration of Helsinki (as revised in 2013). This retrospective cohort study was approved by the institutional review board of the First Affiliated Hospital of Zhejiang University (No.: 2017-712). Informed consent was waived because of the retrospective nature of the study.

Open Access Statement: This is an Open Access article distributed in accordance with the Creative Commons Attribution-NonCommercial-NoDerivs 4.0 International License (CC BY-NC-ND 4.0), which permits the noncommercial replication and distribution of the article with the strict proviso that no changes or edits are made and the original work is properly cited (including links to both the formal publication through the relevant DOI and the license). See: https://creativecommons.org/licenses/by-nc-nd/4.0/.

\section{References}

1. Bosman FT, Carneiro F, Hruban RH, et al. WHO Classification of Tumours of the Digestive System, 4th Edition. Lyon: IARC Press, 2010.

2. Lim JH. Cholangiocarcinoma: morphologic classification according to growth pattern and imaging findings. AJR Am J Roentgenol 2003;181:819-27.

3. Yin X, Zhang BH, Qiu SJ, et al. Combined hepatocellular carcinoma and cholangiocarcinoma: clinical features, treatment modalities, and prognosis. Ann Surg Oncol 2012;19:2869-76.

4. Li Y, Xia Y, Li J, et al. Prognostic Nomograms for Pre- 
and Postoperative Predictions of Long-Term Survival for Patients Who Underwent Liver Resection for Huge Hepatocellular Carcinoma. J Am Coll Surg 2015;221:962-74.e4.

5. Li J, Zhou J, Yang PH, et al. Nomograms for survival prediction in patients undergoing liver resection for hepatitis B virus related early stage hepatocellular carcinoma. Eur J Cancer 2016;62:86-95.

6. Cai MY, Wang FW, Li CP, et al. Prognostic factors affecting postoperative survival of patients with solitary small hepatocellular carcinoma. Chin J Cancer 2016;35:80.

7. Yap AQ, Chen CL, Yong CC, et al. Clinicopathological factors impact the survival outcome following the resection of combined hepatocellular carcinoma and cholangiocarcinoma. Surg Oncol 2013;22:55-60.

8. Jarnagin WR, Weber S, Tickoo SK, et al. Combined hepatocellular and cholangiocarcinoma: demographic, clinical, and prognostic factors. Cancer 2002;94:2040-6.

9. Koh KC, Lee H, Choi MS, et al. Clinicopathologic features and prognosis of combined hepatocellular cholangiocarcinoma. Am J Surg 2005;189:120-5.

10. Yano Y, Yamamoto J, Kosuge T, et al. Combined hepatocellular and cholangiocarcinoma: a clinicopathologic study of 26 resected cases. Jpn J Clin Oncol 2003;33:283-7.

11. Portolani N, Baiocchi GL, Coniglio A, et al. Intrahepatic cholangiocarcinoma and combined hepatocellularcholangiocarcinoma: a Western experience. Ann Surg Oncol 2008;15:1880-90.

12. Kassner A, Thornhill RE. Texture analysis: a review of neurologic MR imaging applications. AJNR Am J Neuroradiol 2010;31:809-16.

13. Ng F, Ganeshan B, Kozarski R, et al. Assessment of primary colorectal cancer heterogeneity by using wholetumor texture analysis: contrast-enhanced CT texture as a biomarker of 5-year survival. Radiology 2013;266:177-84.

14. Huang Y, Liu Z, He L, et al. Radiomics Signature: A Potential Biomarker for the Prediction of Disease-Free Survival in Early-Stage (I or II) Non-Small Cell Lung Cancer. Radiology 2016;281:947-57.

15. Gillies RJ, Kinahan PE, Hricak H. Radiomics: Images Are More than Pictures, They Are Data. Radiology 2016;278:563-77.

16. Maeda T, Adachi E, Kajiyama K, et al. Combined hepatocellular and cholangiocarcinoma: proposed criteria according to cytokeratin expression and analysis of clinicopathologic features. Hum Pathol 1995;26:956-64.

17. Cucchetti A, Piscaglia F, Caturelli E, et al. Comparison of recurrence of hepatocellular carcinoma after resection in patients with cirrhosis to its occurrence in a surveilled cirrhotic population. Ann Surg Oncol 2009;16:413-22.

18. Zhou H, Wang H, Zhou D, et al. Hepatitis B virusassociated intrahepatic cholangiocarcinoma and hepatocellular carcinoma may hold common disease process for carcinogenesis. Eur J Cancer 2010;46:1056-61.

19. Ishikawa K, Sasaki A, Haraguchi N, et al. A case of an alpha-fetoprotein-producing intrahepatic cholangiocarcinoma suggests probable cancer stem cell origin. Oncologist 2007;12:320-4.

20. Ishii T, Yasuchika K, Suemori H, et al. Alpha-fetoprotein producing cells act as cancer progenitor cells in human cholangiocarcinoma. Cancer Lett 2010;294:25-34.

21. Park HS, Bae JS, Jang KY, et al. Clinicopathologic study on combined hepatocellular carcinoma and cholangiocarcinoma: with emphasis on the intermediate cell morphology. J Korean Med Sci 2011;26:1023-30.

22. Asayama Y, Taguchi Ki K, Aishima Si S, et al. The mode of tumour progression in combined hepatocellular carcinoma and cholangiocarcinoma: an immunohistochemical analysis of E-cadherin, alpha-catenin and beta-catenin. Liver 2002;22:43-50.

23. Lee WS, Lee KW, Heo JS, et al. Comparison of combined hepatocellular and cholangiocarcinoma with hepatocellular carcinoma and intrahepatic cholangiocarcinoma. Surg Today 2006;36:892-7.

24. Fleming RE, Ponka P. Iron overload in human disease. $\mathrm{N}$ Engl J Med 2012;366:348-59.

25. Hann HW, Kim CY, London WT, et al. Increased serum ferritin in chronic liver disease: a risk factor for primary hepatocellular carcinoma. Int J Cancer 1989;43:376-9.

26. Uchino K, Tateishi R, Fujiwara N, et al. Impact of serum ferritin level on hepatocarcinogenesis in chronic hepatitis C patients. Hepatol Res 2016;46:259-68.

27. Urano S, Ohara T, Noma K, et al. Iron depletion enhances the effect of sorafenib in hepatocarcinoma. Cancer Biol Ther 2016;17:648-56.

28. Weismuller TJ, Kirchner GI, Scherer MN, et al. Serum ferritin concentration and transferrin saturation before liver transplantation predict decreased long-term recipient survival. Hepatology 2011;54:2114-24.

29. Konomi K, Nakamura K, Tobimatsu M, et al. Identification of carcinoembryonic antigen (CEA) in bile of patients with malignant biliary tract disease. Jpn J Surg 1982;12:184-90.

30. Juntermanns B, Radunz S, Heuer M, et al. Tumor markers as a diagnostic key for hilar cholangiocarcinoma. Eur J 
Med Res 2010;15:357-61.

31. Loosen SH, Roderburg C, Kauertz KL, et al. CEA but not CA19-9 is an independent prognostic factor in patients undergoing resection of cholangiocarcinoma. Sci Rep 2017;7:16975.

32. Dohmen K, Shigematsu H, Irie K, et al. Longer survival in female than male with hepatocellular carcinoma. J Gastroenterol Hepatol 2003;18:267-72.

33. Martin SP, Ruff S, Diggs LP, et al. Tumor grade and sex should influence the utilization of portal lymphadenectomy for early stage intrahepatic cholangiocarcinoma. HPB (Oxford) 2019;21:419-24.

34. Lee JH, Chung GE, Yu SJ, et al. Long-term prognosis of combined hepatocellular and cholangiocarcinoma after curative resection comparison with hepatocellular carcinoma and cholangiocarcinoma. J Clin Gastroenterol 2011;45:69-75.

35. Yang D, Hanna DL, Usher J, et al. Impact of sex on the survival of patients with hepatocellular carcinoma: a Surveillance, Epidemiology, and End Results analysis. Cancer 2014;120:3707-16.

36. Edge SB, Compton CC. The American Joint Committee on Cancer: the 7th edition of the AJCC cancer staging manual and the future of TNM. Ann Surg Oncol 2010;17:1471-4.

37. Park MS, Lee DK, Kim MJ, et al. Preoperative staging accuracy of multidetector row computed tomography for extrahepatic bile duct carcinoma. J Comput Assist Tomogr 2006;30:362-7.

38. Vargas HA, Veeraraghavan H, Micco M, et al. A novel

Cite this article as: Zhang $\mathrm{J}$, Wang $\mathrm{X}$, Zhang L, Yao L, Xue X, Zhang S, Li X, Chen Y, Pang P, Sun D, Xu J, Shi Y, Chen F. Radiomics predict postoperative survival of patients with primary liver cancer with different pathological types. Ann Transl Med 2020;8(13):820. doi: 10.21037/atm-19-4668 representation of inter-site tumour heterogeneity from pre-treatment computed tomography textures classifies ovarian cancers by clinical outcome. Eur Radiol 2017;27:3991-4001.

39. Bae JM, Jeong JY, Lee HY, et al. Pathologic stratification of operable lung adenocarcinoma using radiomics features extracted from dual energy CT images. Oncotarget 2017;8:523-35.

40. Chen X, Wei X, Zhang Z, et al. Differentiation of trueprogression from pseudoprogression in glioblastoma treated with radiation therapy and concomitant temozolomide by GLCM texture analysis of conventional MRI. Clin Imaging 2015;39:775-80.

41. Zhang H, Li W, Hu F, et al. MR texture analysis: potential imaging biomarker for predicting the chemotherapeutic response of patients with colorectal liver metastases. Abdom Radiol (NY) 2019;44:65-71.

42. Huang SY, Franc BL, Harnish RJ, et al. Exploration of PET and MRI radiomic features for decoding breast cancer phenotypes and prognosis. NPJ Breast Cancer 2018;4:24.

43. Cozzi L, Dinapoli N, Fogliata A, et al. Radiomics based analysis to predict local control and survival in hepatocellular carcinoma patients treated with volumetric modulated arc therapy. BMC Cancer 2017;17:829.

44. Lee J, Jain R, Khalil K, et al. Texture Feature Ratios from Relative CBV Maps of Perfusion MRI Are Associated with Patient Survival in Glioblastoma. AJNR Am J Neuroradiol 2016;37:37-43. 

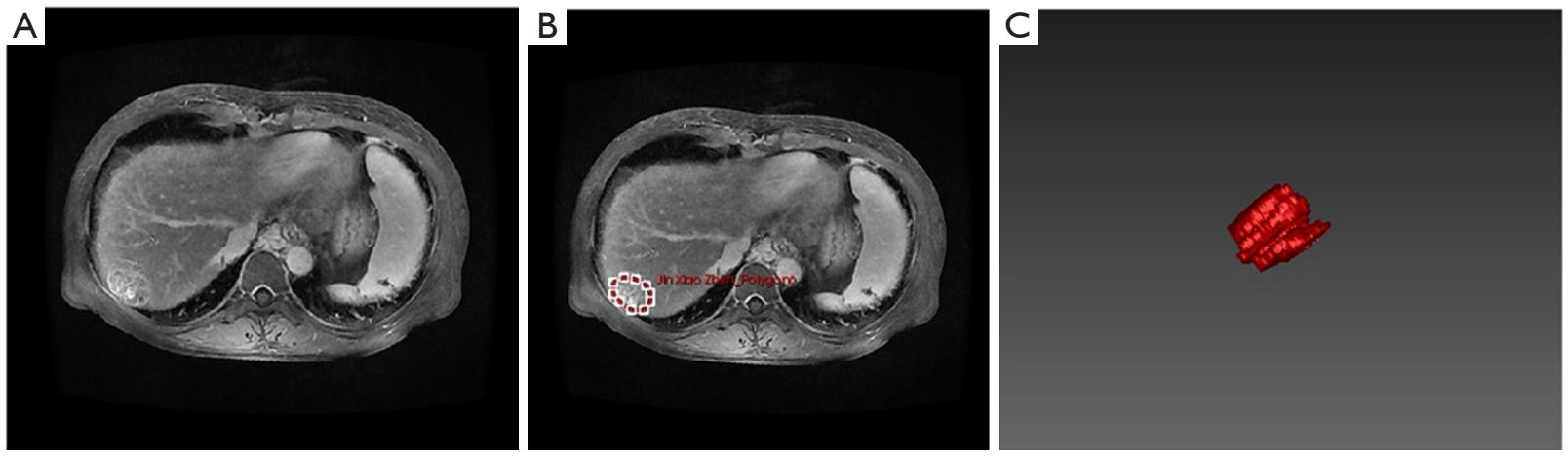

Figure S1 Extraction process of the morphological features. (A) Magnetic resonance enhanced equilibrium phase image; (B) manual delineation of the tumor; $(\mathrm{C})$ three-dimensional segmentation of the lesion. Obtaining the quantitative parameter characteristics.
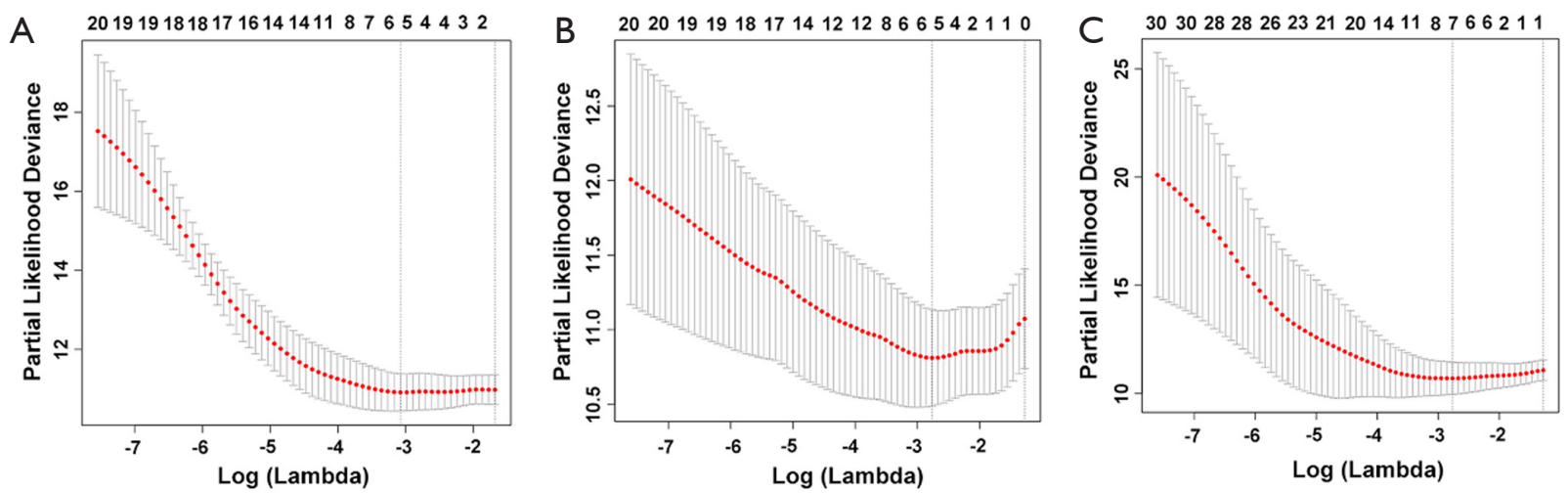

Figure S2 Elastic network dimension reduction diagram. The elastic network method was used to reduce the image feature parameters of the (A) diffusion weighted imaging (DWI), (B) enhanced equilibrium (EP), and (C) DWI and EP. The 5-fold cross validation method was used to determine the optimized lambda $(\lambda)$. The optimized $\lambda$ was $0.04608123,0.06285455$, and $0.06285455[\ln (\lambda):-3.077349,-2.766932$, and -2.766932] and the number of features corresponding to the value is five, five, and seven, respectively. 